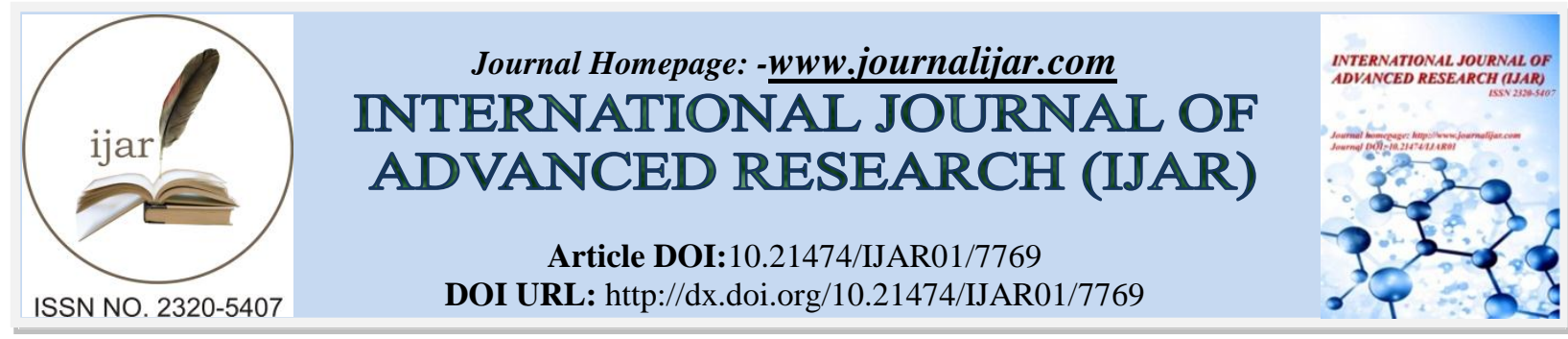

RESEARCH ARTICLE

\title{
EVALUATION THE EFFECT OF THE MALARIA PARASITE ON PROTHROMBIN TIME AND ACTIVATED PARTIAL THROMBOPLASTIN TIME LEVELS AMONG SUDANESE PATIENT IN KHARTOUM STATE- SUDAN.
}

\section{Sarah Mahmoud Abdabagi, Fayhaa Mohammed Altayeb, Ahmed Bakheet Abd Alla,Rania Abd Alrhaman Osman and Ali Elamin Nasir.}

1. Department of Hematology and Immunohematology, College of Medical Laboratory Science, Sudan University of Science and Technology, Khartoum, Sudan.

2. Head Department of Parasitology and Medical Entomology, College of Medical Laboratory Science, Sudan University of Science and Technology, Khartoum, Sudan.

3. Department of Parasitology and Medical Entomology, College of Medical Laboratory Science, Sudan University of Science and Technology, Khartoum, Sudan.

\section{Manuscript Info}

Manuscript History

Received: 25 July 2018

Final Accepted: 31 August 2018

Published: September 2018

Keywords:-

PT, aPTT, Malaria, hemostasis.

\section{Abstract}

The hemostatic alterations play an important role in malaria pathogenesis and in the progression of disease. In severe infection the accelerated coagulation cascade activity with accelerated fibrinogen turnover increase the bleeding tendency and may lead to DIC. The present study was aimed to determine the effect of malaria parasites on Prothrombin Time and Activated Partial Thromboplastin Time Levels. A single citrated blood sample of patients diagnosed with malaria was analysed in semi- automated coagulation analyzer at the time of presentation, the values were compared with healthy controls. Data was analyzed by mean standard deviation and by ' $t$ ' test using SPSS software version 16. PT and APTT were significantly raised in cases when compared to control group, prolonged APTT is observed in $44 \%$ (P.value.001) and prolonged PT is observed in $43 \%$ of cases (P. value $.016)$, the mean of PT $(15.73 \mathrm{sec})$, and the mean of APTT $(40.24 \mathrm{sec})$ in cases group. The mean of PT and APTT in control group, (13.94 sec), $(30.87 \mathrm{sec})$ respectively. This study concluded that malaria infection altered the blood coagulation mechanism causing significant prolongation in PT and APTT.

Copy Right, IJAR, 2018,. All rights reserved.

\section{Introduction:-}

Normal homeostasis is a series of regulated processes that maintain blood in clot-free state in normal vessels while rapidly forming a localized plug at the site of vascular injury (1). The haemostatic response to vascular damage depends on the endothelium cells, platelets and the coagulation proteins which include 3 protein systems: the clotting factors, the fibrinolytic and anticoagulant factors (2). Malaria is one of the most deadly and epidemic diseases that affects Sudan according to the latest WHO data published in 2016 the reported confirmed cases are 575,015 and the reported deaths are 984(3). Malaria infection is considered one of the Formatting Your Paper acquired bleeding diathesis in which many disturbances in the blood coagulation system contribute to the inflammation and organ failure associated with severe disease (4)(5). Factors contributing to complication in malaria 
include sequestration of parasitized RBCs in microcirculation, activation of inflammation with production of cytokine storm, widespread activation of coagulation system accompanied by formation of thrombi in microvascular bed producing multi-organ dysfunction and disseminated intravascular coagulation (DIC) (6). The haemostatic alterations due to the presence of the parasite in blood stream can be triggered by activation of endothelial cells, which causes release of ultra large multimers of Von-Willebrand factor which together with deficiency of A Disinterring And Metalloproteinase with a Thrombo-Spondin 13 (ADAMTS13) in affected individuals causes platelet adhesion and aggregation and release of procoagulant substances (7)(8). Also decreased levels of plasma antithrombin or elevated levels of plasminogen activator inhibitor (PAI)-1, and thrombocytopenia are frequently found (9)(10). The infected RBCs also induce tissue factor expression by endothelial cells and support the assembly of coagulation complexes, the thrombin generated can activate platelets, produce microthrombi and activate the intrinsic pathway of coagulation(11), it also releases cytokines in to bloodstream which are procoagulant material lead to propagation of coagulation cascade (12). In addition, TF, thrombin and activated FX together can trigger the inflammatory system by enhancing the production of cytokines especially interleukins and Tumor Necrosis Factor(13)(14). In uncomplicated malaria Most of the cases remain in a state of compensated DIC when the coagulation disorder is a common laboratory finding with a recognized prolongation of prothrombin time and APTT, but bleeding and hemorrhage are not observed, typical DIC is observed in severe malaria when the continued presence of the parasite leads to consumption of the anticoagulants which are then no longer available to manage the activated factors leading to large scale thrombosis (13)(15).

\section{Materials and Methods:- \\ Study design}

This study was cross-sectional case control and hospital base study.

\section{Study area and duration}

The study was carried out in the Military hospital, Al-Ameen Hamed Hospital, Tropical Disease Hospital and ALbalsam Clinic in Khartoum State, it was Conducted in period between April and August 2017.

\section{Study population}

A total of 50 patients with different ages, who were diagnosed with malaria infection and 50 healthy volunteers as a control group were enrolled in this study.

\section{Inclusion Criteria}

Cases included only fully diagnosed malaria patients, while Controls included only apparently healthy individual.

\section{Exclusion Criteria}

Malaria patients under treatment, Malaria patient with other diseases, Patients on anticoagulant therapy, known disease states having abnormal coagulation profiles like hemophilia and Pregnant women were excluded.

\section{Data collection}

Data were collected using non-self-administered questionnaires. The questionnaires were specifically designed to collect demographic data about patient ID number, age, sex, and method by which patient diagnosed.

\section{Data analysis}

Statistical analyses were performed by using a computer based statistical program SPSS (Statistical Package for Social Science), version 16.0.

\section{Sample collection}

A $2.7 \mathrm{ml}$ of venous blood to $0.3 \mathrm{ml}$ of $3.2 \%$ tri sodium citrate was collected from patient/control using disposable sterile syringe after disinfecting collection site with $70 \%$ alcohol.

\section{Preparation of platelet poor plasma}

PPP was prepared by centrifugation of the citrated sample at $1500 \mathrm{rpm}$ for 15 minutes then the plasma transfused in to plane container. 


\section{Measuring of PT}

The test was done by using of a semiautomated Coagulometer (Dia timer 2), the Cuvettes were placed in incubation area for pre-warming at $37 \mathrm{c}$ for 3 minutes. A $100 \mathrm{ul}$ of pre-wormed control or patient PPP was dispensed in cuvette in incubation area. Then cuvettes transferred to test area and 200ul of well mixed calcified thromboplastin reagent were added to cuvette, the analyzer timer started automatically when reagent was added. When clot formed timer stopped automatically as result of optical density changes, the analyzer is bi channel, get the mean of the two tested cuvettes and express it as PT on instrument display screen per seconds together with calculated INR.

\section{Measuring of APTT}

In Dia timer 2 the Cuvettes were placed in incubation area for pre-warming at $37 \mathrm{c}$ for 3 minutes.A $100 \mathrm{ul}$ of prewormedcontrol or patient PPP was dispensed in cuvette in incubation area. Then a $100 \mathrm{ul}$ of cephalin/ kaolin mixture was added to each cuvette. After 3-minute incubation 100ul of calcium chloride were added to each cuvette after they transferred to test area. The mean of the two tested cuvettes is expressed as APTT on instrument display screen per seconds.

\section{Results:-}

The Comparison between the PT and APTT values of malaria patients and healthy controls were significantly different, the mean of PT in cases group is $(15.736 \pm 1.8510 \mathrm{sec})$ and the mean of APTT is $(40.242 \pm 8.0779 \mathrm{sec})$, while the means of PT and APTT in controls group are $(13.948 \pm 1.2770 \mathrm{sec})$ and $(30.870 \pm 3.8311 \mathrm{sec})$ respectively. The prolongation in APTT is highly significant (P.value 0.001 ) observed in $44 \%$ of patients, and the prolongation in PT (P. value .016) is observed in $43 \%$ of malaria patients (Table1).

Fifty malaria-infected subjects were included in this study, out of these, $26(52 \%)$ were males and 24 (48\%) females, the mean of PT in males is $(15.88 \pm 1.896 \mathrm{sec})$ and in females is $(15.58 \pm 1.827 \mathrm{sec})$ and the mean of APTT in males is $(38.54 \pm 6.64 \mathrm{sec})$ and in females is $(42.08 \pm 9.18 \mathrm{sec})$ in the cases group, the study shows that there is no significant differences between genders in PT and APTT (P.value for PT $=0.685$ and P.value for APTT=0.394) (Table2).

The patients were subdivided in to three age groups, less than 5 years with a mean of age (3.5) and the means of PT and APTT were $(15.875 \pm 1.41)(48.075 \pm 4.854)$ respectively, from 5 through 15 the a mean age is $(8.9)$ and the means of PT and APTT were (15.445 \pm 2.011$)(43.191 \pm 4.877)$ and over 15 years with a mean age of (33.2) the means of PT and APTT were(15.671 \pm 1.963$)(38.657 \pm 8.726)$, there is no significant differences between different age groups in cases (P.value for PT $=0.305$, P.value for APTT $=0.511)$ (Table3).

Table (1):-The comparison between the mean of PT and APTT in cases and control

\begin{tabular}{|c|c|c|c|}
\hline & Cases & Control & P-value \\
\hline PT & $15.736 \pm 1.8510$ & $13.948 \pm 1.2770$ & .016 \\
\hline APTT & $40.242 \pm 8.0779$ & $30.870 \pm 3.8311$ & .001 \\
\hline
\end{tabular}

Table (2):-The comparison between the mean of PT and APTT in cases according to sex

\begin{tabular}{|l|l|l|l|}
\hline \multirow{2}{*}{} & \multicolumn{2}{|c|}{ Patients } & \multirow{2}{*}{ P.value of patients } \\
\cline { 2 - 4 } & Male & Female & \\
\hline PT & $15.88 \pm 1.896$ & $15.58 \pm 1.827$ & 0.685 \\
\hline APTT & $38.54 \pm 6.64$ & $42.08 \pm 9.18$ & 0.394 \\
\hline
\end{tabular}

Table (3):-The comparison between the mean of PT and APTT in cases according to age groups

\begin{tabular}{|c|c|c|c|c|}
\hline \multirow{2}{*}{} & \multicolumn{3}{|c|}{ Patients } & \multirow{2}{*}{ P. value } \\
\cline { 2 - 4 } & $>5$ & $5-15$ & $>18$ & \\
\hline PT & $15.875 \pm 1.41$ & $15.445 \pm 2.011$ & $15.671 \pm 1.963$ & 0.305 \\
\hline APTT & $48.075 \pm 4.854$ & $43.191 \pm 4.877$ & $38.657 \pm 8.726$ & 0.511 \\
\hline
\end{tabular}

\section{Discussion:-}

The present study showed that the coagulation potential of infected patients, measured by an in vitro hemostatic tests PT and APTT, was significantly elevated, reflecting a procoagulant status. This indicates that in patient with malaria 
there is an activation of intrinsic and extrinsic pathways and the coagulation system reacts early during infection which leads to prolonged PT and PTT (15) (16). These results are compatible to the study carried out by Reshma et al., in India (2016) who find that the difference in the coagulation profile of malaria was significant in vivax malaria to the same extent as falciparum malaria., the PT and APTT were prolonged in $38 \%$ and $56 \%$ of the malaria patients ( $\mathrm{P}<0.001$ in both parameters) (15). Other study done by Das et al., (2011) Showed prolonged PT, prolonged APTT in $80 \%, 70 \%$ out of 40 cases ( $\mathrm{P}<0.05$ in both parameters) (17). Rojanasthein et al. (1992) also revealed significantly prolonged PT (47.5\%) and APTT (35\%) values in patient with P.falciparum malaria but no difference in values in patients with vivax malaria (18). Malaria parasite possesses several different mechanisms capable of affecting the tightly balanced activities of procoagulant and anticoagulant factors that maintain homeostasis. These results suggests that the haemostatic dysfunction plays a key role in the pathogenesis of malaria, and might have a role in advanced infection and the development of complications due to the deplete ion of various factors in the cascade that proceed to hypo coagulable condition which further leads to hemorrhagic manifestations(19). Correlating the PT and APTT values with the age and gender, showed no significant differences in PT and APTT values between genders or different age groups, this is indicate that gender and age does not affect the mechanisms by which malaria alter the normal coagulation process.

\section{References:-}

1. Kumar, v., Abbas, k., and Aster, j. (2013). Robbins basic pathology. 9th ed. Canada: Elsevier Saunders, p84

2. Schmaier, A. H., and Lazarus, H. M. (2012). Concise Guide to Hematology. 1th ed. UK: Blackwell Publishing Ltd, p 97, 98, 99, 100, 101.

3. World Health Organization. (2016). World Malaria Report (2016), Available at www.who.int/malaria/countries/sdn/en/.

4. Francischetti, I. M., Seydel, K. B. and Monteiro, R. Q. (2008). Blood coagulation, inflammation, and malaria.Microcirculation, 15(2):81-107

5. Francischetti, I. M. (2008). Does activation of the blood coagulation cascade have a role in malaria pathogenesis?Trends Parasitol.;24(6):258-263.

6. Miller, L. H., Baruch, D. I., Marsh, K. and Doumbo, O. K. (2002). The pathogenic basis of malaria, NATURE; 415: 673-674

7. Graham, S. M., Chen, J., Chung, D.W. and Barker, K. R. et al., (2016). Endothelial activation, haemostasis and thrombosis biomarkers in Ugandan children with severe malaria participating in a clinical trial. Malaria journal, 15:56.

8. De Mast, Q., Groot, E., Asih, P. B., Syafruddin, D., Oosting, M., Sebastian, S., ... and Fijnheer, R. (2009). ADAMTS13 deficiency with elevated levels of ultra-large and active von Willebrand factor in P. falciparum and P. vivax malaria. The American journal of tropical medicine and hygiene, 80(3), 492-498.

9. Akinosoglou KS, Solomou EE, Gogos CA. (2012). Malaria: a haematological disease. Hematol Amst Neth;17:10614. doi:10.1179/1024533.

10. Angchaisuksiri P. (2014). Coagulopathy in malaria,Thrombosis Research; 133: 5-9

11. Mohanty, D., Marwaha, N., Ghosh, K., Sharma, S., Garewal, G., Shah, S., Devi, S. and Das, K. C. (1988). Functional and ultrastructural changes of platelets in malarial infection. Trans R Soc Trop Med Hyg;82:369.

12. Francischetti, N. B., Seydel. K. B. and Monteiro, R. Q. (2005). Critical role of blood coagulation in malaria. Blood journal, 106(11):4044.

13. Francischetti, N. B., Seydel, K. B. and Monteiro, R.Q. (2008). Blood coagulation, Inflammation and malaria. Informa healthcare, 15: 81-107.

14. Francischetti, I. M. B., Seydel, K. B., Monteiro, R. Q., Whitten, R. O., Erexson, C. R., Noronha, A. L. L., Ostera, G. R., Kamiza, S. B., Molyneux, M. E., Ward, J. M. and Taylor, T. E. (2007). Plasmodium falciparum-infected erythrocytes induce tissue factor expression in endothelial cells and support the assembly of multimolecular coagulation complexes. J Thromb Haemost; 5: 155-65.

15. Reshma, K., Veronica, L., Raphael, L., and Vedasree, M. (2016). Clinical Correlation of Coagulopathy in Vivax Malaria, Pacific Group of e-Journals, 3(1): 61-65.

16. Ried, j., Mordmuller, B., Koder, S., Hoffman, S. L. and Kremsner, P. G. (2016). Alterations of blood coagulation in controlled human malaria infection. Malaria journal. 15: 15.

17. Das, S., Datta, S., Roul, L. D., Panda, J. K., Pattanayak, N. C., and Bhattacherjee, s. (2011). A study of the coagulation profile in falciparum malaria, Orissa physicians journal, 7: 7-12.

18. Rojanasthein, S., Surakamolleart, V., Boonpucknavig, S., and Isarangkura, P. (1992). Hematological and Coagulational Studies, Malaria journal, 75(1):190- 194.

19. Mohapatra, S., Samantary, J.C., Arulselvi, S., and Ghosh, A. (2013). Disseminated intravascular coagulation following malaria due to Plasmodium vivax, Malaria journal, 12: 336. 